\title{
Um dashboard para a percepção dos professores sobre as atividades de estudantes em ambiente virtual de aprendizagem
}

Carolina V. Costa Gomes, UNIVASF, carolinagomes08@gmail.com, https://orcid.org/0000-0003-2132-8446 Jorge Luis. C. Ramos, UNIVASF, jorge.cavalcanti@nivasf.edu.br, https://orcid.org 0000-0002-6099-6861 João Carlos. Sedraz Silva, UNIVASF joao.sedraz@univasf.edu.br, https://orcid.org 0000-0002-4082-9652 Rodrigo Lins Rodrigues, UFRPE, rodrigo.linsrodrigues@ufrpe.br, https://orcid.org 0000-0002-3598-5204

Resumo. Este estudo apresenta o desenvolvimento de um dashboard para ser incorporado a um ambiente virtual de aprendizagem. $O$ dashboard permite aos professores um maior acompanhamento das atividades dos estudantes no ambiente. As diretrizes da pesquisa foram definidas a partir da análise de três outras propostas similares, sendo o método Design Science Research escolhido para o percurso metodológico. Os resultados apontam que o dashboard obteve bons indicadores, tanto para questões de usabilidade quanto para aceitação do artefato proposto.

Palavras-chave: Learning Analytics, Amadeus LMS, Design Science Research, DSR.

\section{A dashboard for teachers' perception of student activities in a virtual learning environment}

\begin{abstract}
This study presents the development of a proposed dashboard to be incorporated into a virtual learning environment. The dashboard should allow teachers to better monitor student activities in the learning environment. The research guidelines were defined based on the analysis of three other similar proposals, the Design Science Research method being chosen for the methodological path. The results show that the dashboard obtained good indicators, both for usability issues and for the acceptance of the proposed artifact.
\end{abstract}

Keywords: Learning Analytics, Amadeus LMS, Design Science Research, DSR.

\section{Introdução}

É notório o crescimento de cursos que utilizam a abordagem de educação a distância (EAD), ou até mesmo, atividades remotas como complemento de carga horária em cursos presenciais (blended learning). Tal crescimento pode ser justificado pelo aumento de ferramentas tecnológicas inseridas no contexto socioeconômico e cultural dos estudantes (TORI, 2009).

Entretanto, com distanciamento físico entre alunos e professores, fica ainda mais difícil a percepção de lacunas na aprendizagem, bem como suas causas, uma vez que não está ao alcance do professor a observação, controle, e ajuste do processo educacional (VOZNIUK; GOVAERTS; GILLET, 2013).

A visualização de dados educacionais tem sido uma importante área de pesquisa e desenvolvimento de soluções complementares aos processos de mineração de dados educacionais e Learning Analytics (LA).

Nesse contexto, este trabalho propôs o emprego de um dashboard para professores universitários, para acompanhamento de atividades realizadas no ambiente virtual de aprendizagem Amadeus LMS (do inglês Learning Management System) em um contexto de blended learning. Além disso, foram avaliadas a usabilidade e aceitação dos recursos implementados com usuários. Um dashboard é um painel visual contendo uma série de elementos gráficos que facilitam a visualização e análise de dados.

Para tanto, foi realizada inicialmente, uma revisão bibliográfica e uma análise de trabalhos relacionados, para investigar os aspectos mais relevantes na implementação de soluções de Learning Analytics (LA) voltadas para o uso de professores. Em seguida, foram 
desenvolvidas versões preliminares do painel, com o intuito de planejar e organizar a integração dos aspectos investigados na solução de LA proposta. Após isso, foi implementado um protótipo funcional no Amadeus LMS, com todas os recursos ativados e já disponíveis para os testes.

O protótipo funcional foi colocado em uso com 10 professores da Universidade Federal do Vale do São Francisco (UNIVASF). Cada participante da pesquisa realizou um roteiro de testes e, em seguida, avaliou o dashboard em relação à usabilidade e aceitação. Foram obtidos resultados favoráveis em ambos os aspectos.

Assim, este trabalho demonstra a importância da implantação de soluções de LA no contexto educacional, além de propor uma destas soluções para um Software Público Brasileiro, contribuindo com a comunidade de softwares livres e públicos.

\section{Fundamentos teóricos}

Os principais conceitos envolvidos neste estudo são apresentados nos tópicos a seguir.

\subsection{Learning Analytics}

A Learning Analytics é uma área de pesquisa que busca o desenvolvimento de soluções tecnológicas no âmbito educacional, através da medição, coleta, análise, e comunicação de dados sobre estudantes, a fim de fornecer uma melhor compreensão de como o processo de aprendizagem está ocorrendo [Siemens et al., 2011].

Picciano (2014) definiu o termo Learning Analytics como a ciência de examinar dados, a fim de deduzir conclusões que culminam na tomada de decisão por um caminho ou curso de ações. No contexto educacional, essa análise tem sido usada no monitoramento dos alunos para aumentar a eficiência dos processos de aprendizagem.

A Society for Learning Analytics Research (SoLAR - https://solaresearch.org/), estabeleceu que a Learning Analytics se caracteriza pela medição, coleta, análise e comunicação de dados sobre os estudantes e seus contextos, para compreensão e otimização da aprendizagem e do ambiente em que ela ocorre [Siemens et al., 2011].

Clow (2012) propôs uma forma sistemática de visualizar o processo de Learning Analytics (Figura 1), que inclui estudantes, dados, análise e intervenções.

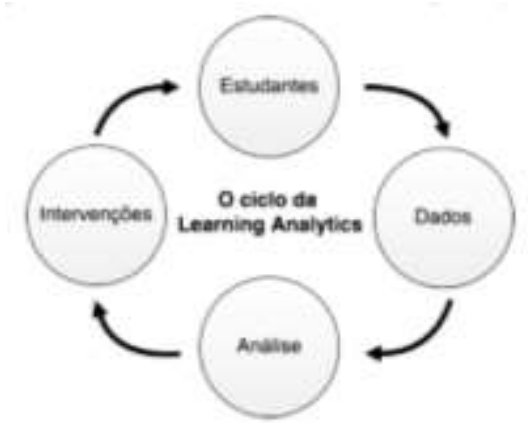

Figura 1 - 0 ciclo da Learning Analytics. Fonte: Silva (2018), adaptado de Clow(2012).

A primeira etapa do ciclo, é a definição do conjunto que irá compor a análise, que deve ser composto por estudantes. A próxima etapa, é a geração e a captura de dados sobre os estudantes, a partir das interações em AVAs e dos resultados de avaliações.

A terceira etapa do ciclo, consiste no processamento dos dados a fim de transformálos em informações úteis, que podem ser exibidos em forma de visualizações, dashboard e resultados de análises comparativas realizadas por benchmarks. Clow (2012) considerou este o passo principal no ciclo proposto, sendo o foco principal nos projetos de LA.

Por fim, o ciclo se completa quando as informações geradas na terceira etapa são usadas para gerar intervenções no processo de aprendizagem, que tenham efeito sobre os estudantes. $\mathrm{O}$ autor destacou, entretanto, que um projeto de LA não precisa, necessariamente, ser composto por todas as etapas do ciclo proposto. 


\subsection{Visualização de Dados Educacionais}

A visualização de dados educacionais compreende uma das formas mais efetivas de aplicação da Learning Analytics, uma vez que propõe o uso de ferramentas interativas para o uso no meio acadêmico, transmitindo as informações acerca dos estudantes de forma muito mais dinâmica e intuitiva [Silva et al., 2016; Chatti et al., 2013].

A visualização do progresso em direção a uma meta, é uma forma efetiva de alcançála. Tanto para estudantes quanto para professores, é útil ter uma visão geral do andamento de atividades e de como isso se relaciona com o processo educacional [Duval, 2011].

Chatti et al. (2013) destacaram que relatórios com dados estatísticos e tabelas de dados, nem sempre são muito fáceis de serem interpretados. Porém, a implementação dos métodos da LA em uma ferramenta visual, pode facilitar muito a análise de dados educacionais, por parte dos usuários, tornando o processo mais intuitivo.

No contexto da LA, as ferramentas de visualização de dados podem assumir vários papéis. Do ponto de vista dos alunos, podem ser utilizadas para monitoramento de seu desempenho escolar. Para os professores, podem ser úteis para acompanhamento das turmas em tempo real, permitindo a identificação de padrões positivos e negativos, que podem indicar falhas e acertos nos métodos pedagógicos utilizados. Além disso, pode auxiliar na identificação de estudantes de alto rendimento, ou em situação de risco, possibilitando que sejam feitas intervenções a tempo [Chatti, 2013].

Um dos maiores problemas nas ferramentas visuais de LA, entretanto, é a incerteza sobre quais os dados que devem ser medidos, a fim de fornecer indicadores úteis. Geralmente, essas medidas incluem: o tempo gasto em uma tarefa, o número de vezes que um usuário acessou o sistema, a quantidade de cliques com o mouse, entre outras variáveis. O problema real está em determinar a efetividade destas informações [Duval, 2011].

\section{Trabalhos Relacionados}

Este tópico apresenta três trabalhos existentes na literatura sobre estratégias e funcionalidades mais utilizadas para implementação de dashboards educacionais direcionados ao uso de professores, bem como a efetividade dos mesmos.

\subsection{LOOP}

Corrin et al. (2015) desenvolveram como uma solução de LA para professores, o LOOP, um dashboard que fornece uma visão geral do andamento das atividades oriundas de um determinado curso. O LOOP é uma ferramenta de código aberto, que foi implementada com intenção de uso nos ambientes LMS Moodle e Blackboard.

O dashboard tem diferentes áreas de visualizações. Uma delas contém as interações diárias nas visitas à página da disciplina em que o dashboard está sendo utilizado.

Em outra seção, linhas verticais de um gráfico permitem a identificação, por parte dos professores, de períodos críticos. Esta representação visual permite que os professores percebam facilmente como a interação dos estudantes com os materiais do curso afetam (ou não) a ocorrência de períodos críticos. Por exemplo, com a inserção de uma atividade avaliativa no ambiente, é esperado que haja um pico de acessos por parte dos alunos.

A representação de métricas semanais indica um resumo de movimentações referentes a algumas atividades no curso, incluindo: os números de visualizações da página, o número de estudantes que estão ativos, o número médio de sessões, e o número médio de páginas vistas por sessão. O painel também mostra a lista de materiais mais acessados do curso, no período de tempo selecionado pelo usuário.

\subsection{Moodle Analytics Dashboard}

Einhardt, Tavares e Cechinel (2016) desenvolveram o Moodle Analytics Dashboard (MAD). O MAD é uma extensão para o navegador Google Chrome, e foi desenvolvida para uso no 
ambiente LMS Moodle. Uma vez instalada pelo usuário no navegador, o MAD é iniciado no momento em que o usuário está em um ambiente Moodle.

A interface do MAD possui um gráfico da parte superior da imagem, com um diagrama de dispersão, onde o usuário pode selecionar duas métricas e relacionar uma com a outra. Em outra área, fica disponível uma lista de participantes do curso, onde o usuário pode selecionar um nome e enviar uma mensagem para o mesmo. Um gráfico na parte inferior, indica a quantidade de acessos à página do curso pelos alunos ao longo do tempo.

Além disso, o MAD fornece também, uma visualização que informa os níveis de acesso de atividades, páginas e alunos, através de um gráfico de barras. A informação a ser impressa no gráfico deve ser selecionada pelo usuário. Essa visualização permite a identificação de alguns padrões, como alunos que estão abaixo ou acima da média de interações da turma.

\subsection{Luna}

Xhakaj, Aleven e McLaren (2017) desenvolveram um protótipo de alta fidelidade de um dashboard para professores, o Luna, para uso em um Sistema de Tutoria Inteligente. O principal objetivo do dashboard consistiu em reportar dificuldades encontradas pelos alunos, aos professores, auxiliando-os na preparação das aulas seguintes, focando nos pontos onde foram identificadas maiores dificuldades.

Para níveis de demonstração e avaliação, o Luna foi posto em uso em oito turmas de ensino fundamental. O Luna foi avaliado em relação aos seus efeitos sobre a tomada de conhecimento dos professores sobre os níveis de aprendizagem dos alunos, e sobre as mudanças que ocorreram no planejamento de aulas dos professores. Foi analisado também, como essas mudanças afetaram o desempenho dos alunos.

Fica claro, pela análise dos trabalhos mostrados nesta seção, que cada dashboard apresentou um direcionamento diferente. $\mathrm{O}$ dashboard Luna foi desenvolvido para medir o desempenho dos alunos em relação às atividades realizadas no ambiente virtual Lynnette.

O MAD, preocupou-se principalmente, em mostrar estatísticas referentes aos acessos à página do curso no ambiente Moodle, e à interação entre os alunos em fóruns de discussão.

O LOOP, por sua vez, apresenta visualizações referentes aos acessos, às interações entre os alunos em fóruns, e ao desempenho dos estudantes em avaliações, uma vez que disponibiliza tabelas com as notas dos alunos.

O Quadro 1 sumariza as características encontradas nos três trabalhos relacionados apresentados, fornecendo diretrizes para a implementação do dashboard a ser apresentado.

Quadro 1 - Resumo da análise de trabalhos relacionados.

\begin{tabular}{|c|c|c|}
\hline \multirow{2}{*}{ Dimensão } & \multicolumn{2}{|c|}{ Elementos identificados nos trabalhos relacionados } \\
\cline { 2 - 3 } & Características relevantes & Referência \\
\hline \multirow{3}{*}{ Dados } & Dados de sistema de tutoria inteligente & Xhakaj, Aleven e McLaren (2017) \\
\cline { 2 - 3 } & Dados de LMS & $\begin{array}{c}\text { Corrin et al. (2015), Einhardt, Tavares e } \\
\text { Cechinel (2016) }\end{array}$ \\
\hline \multirow{3}{*}{ Instrumentos } & Tabelas de dados & Corrin et al. (2015) \\
\cline { 2 - 3 } & Visualização de dados & $\begin{array}{c}\text { Corrin et al. (2015), Einhardt, Tavares e } \\
\text { Cechinel (2016) Xhakaj, Aleven e McLaren } \\
\text { (2017) }\end{array}$ \\
\hline \multirow{3}{*}{ Limitações internas } & Avaliação de aceitação & \begin{tabular}{c} 
Corrin et al. (2015) \\
\cline { 2 - 3 }
\end{tabular} \\
\cline { 2 - 3 } & Avaliação de usabilidade & $\begin{array}{c}\text { Corrin et al. (2015), Einhardt, Tavares e } \\
\text { Cechinel (2016) }\end{array}$ \\
\cline { 2 - 3 } & $\begin{array}{c}\text { Medidas dos efeitos sobre mudanças de } \\
\text { comportamento nos clientes dos dados }\end{array}$ & Xhakaj, Aleven e McLaren (2017) \\
\hline
\end{tabular}

Essas dimensões e características relevantes foram levadas em consideração como requisitos e guiaram o desenvolvimento e a avaliação do dashboard. 


\section{Percurso Metodológico}

Nesta seção, serão apresentadas as diretrizes que nortearam a pesquisa, assim como o método utilizado, com a descrição sumária de cada etapa.

A partir da análise dos trabalhos relacionados, foram definidas como diretrizes da pesquisa, algumas características a serem implementadas no dashboard para professores. Estas características são indicadas no Quadro 2.

Quadro 2 - Métricas a serem implementadas.

\begin{tabular}{|c|c|}
\hline Característica & Descrição \\
\hline Seleção do período & $\begin{array}{l}\text { O usuário deverá ser capaz de selecionar o período sobre o qual os dados serão } \\
\text { mostrados. }\end{array}$ \\
\hline Visitas à página & $\begin{array}{c}\text { Visualização referente ao número de visitas que a página da disciplina possui, } \\
\text { distribuídas ao longo do tempo, de forma que o usuário possa identificar períodos de } \\
\text { tempo críticos (com muitos ou com poucos acessos). }\end{array}$ \\
\hline $\begin{array}{l}\text { Ranking de participantes com } \\
\text { mais acessos }\end{array}$ & $\begin{array}{c}\text { Visualização que permita comparar participantes que se sobressaem em relação a outros } \\
\text { e em relação aos acessos, e, consequentemente, ao engajamento na disciplina. }\end{array}$ \\
\hline $\begin{array}{l}\text { Tópicos e recursos com mais } \\
\text { acessos }\end{array}$ & $\begin{array}{l}\text { Visualização referente ao número de acessos a cada tópico (ou tags no caso do } \\
\text { Amadeus), e a cada recurso disponível, no ambiente da disciplina. }\end{array}$ \\
\hline
\end{tabular}

\subsection{Caracterização da pesquisa}

O método desta pesquisa é orientado pelo paradigma Design Science. Nesse paradigma a geração de conhecimento ocorre a partir da produção de artefatos que contribuam na solução de problemas [Bax, 2017]. É essencial, entretanto, que juntamente com o desenvolvimento de tais artefatos sejam comprovadas a utilidade e a relevância dos mesmos [Silva, 2018].

Peffers et al. (2007) desenvolveram o Design Science Research, um método no qual o projeto de uma pesquisa dirigida ao desenvolvimento de artefatos é organizado em seis etapas, conforme mostrado na Figura 2.

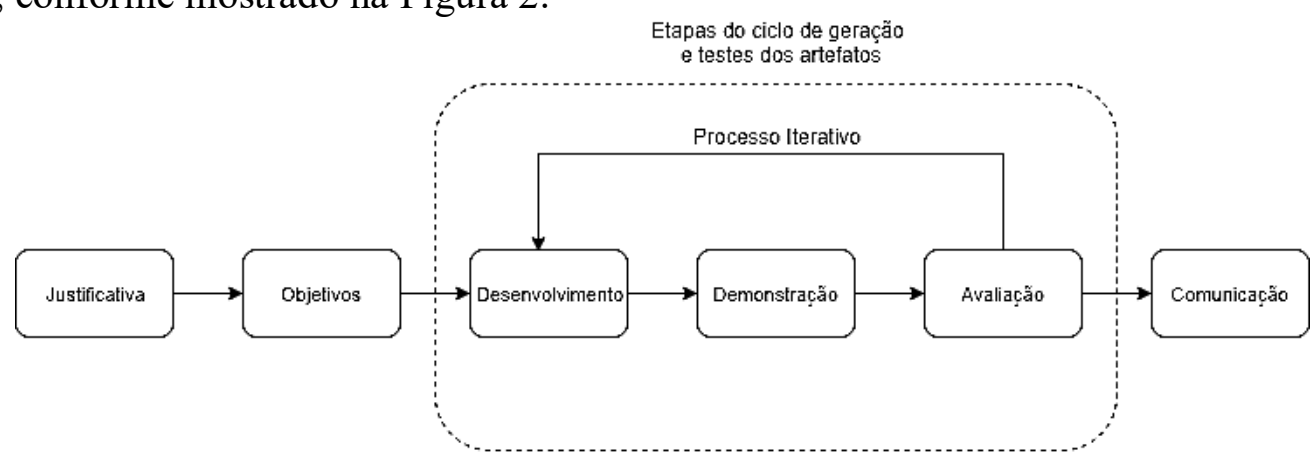

Figura 2 - Etapas do Design Science Research. Fonte: Adaptado de Peffers et al. (2007).

De acordo com o Design Science Research, primeiramente, deve-se apresentar a justificativa para a realização da pesquisa, ou seja, aquilo que a motivou. Em seguida, devem ser definidos os objetivos que se deseja alcançar com o resultado do trabalho. Depois, o projeto entra em um processo, no qual o artefato é desenvolvido, demonstrado e avaliado. Cabe ao autor da pesquisa, decidir se após a avaliação, é viável retornar à etapa de desenvolvimento, a fim de implementar melhorias no artefato. $\mathrm{Na}$ etapa de comunicação, devem ser descritos, detalhadamente, os passos que conduziram a pesquisa e seus resultados [Peffers et al., 2007].

Para este trabalho, as etapas de "Justificativa" e "Objetivos" foram apresentadas na Seção 1. A etapa de "Desenvolvimento" aconteceu a partir da revisão da literatura e da análise de trabalhos relacionados, onde foram estabelecidas as diretrizes da pesquisa e os critérios para elaboração do dashboard. Ainda nesta etapa, foram desenvolvidos os protótipos em baixa e alta fidelidade para as etapas seguintes.

Na etapa de "Demonstração", os protótipos foram apresentados aos professores, para a conhecimento e posterior realização de testes roteirizados. Dumas e Redish (1999), defendem que uma amostra de 5 a 12 usuários é suficiente para testar um sistema 
computacional, uma vez que possíveis problemas de usabilidade encontrados com estes participantes, não irão variar muito quando expostos a um número maior de usuários.

Dessa forma, o experimento foi realizado com 10 professores da UNIVASF. O perfil dos mesmos foi determinado por meio das respostas ao um questionário de avaliação dos protótipos. Assim, a fase de "Avaliação" consistiu em uma investigação dos feedbacks fornecidos pelos professores, em testes de usabilidade e aceitação aplicados. Cada professor executou um roteiro previamente ajustado, com tarefas que exploraram as diversas funcionalidades do dashboard.

Os dados para a avaliação de usabilidade do artefato foram coletados por meio da aplicação do questionário System Usability Scale (SUS) [Brooke, 1996], amplamente utilizado para testes semelhantes, por poder ser aplicado de forma simples, com alto nível de confiabilidade em seus resultados, mesmo quando aplicado a poucos participantes, e por contemplar diversos aspectos da usabilidade.

Além disso, foram acrescentadas ao formulário, questões que buscaram medir o nível de aceitação, tanto em relação à intenção de uso, quanto em relação ao visual do dashboard. Essas questões foram baseadas no Modelo de Aceitação de Tecnologia (Technology Acceptance Model- TAM) [Davis; Bagozzi; Warshaw, 1989].

Por fim, a etapa de "Comunicação", refere-se aos resultados que serão apresentados a seguir, no capítulo seguinte deste estudo.

\section{Resultados e Discussões}

Nesta seção são apresentados e discutidos os resultados da pesquisa, conduzida conforme o método descrito anteriormente. Por conta da limitação do tamanho do texto, serão destacados somente os principais resultados, sendo toda a descrição dos demais resultados disponibilizados em Gomes (2020).

Antes de prosseguir com a definição das visualizações escolhidas, é válido ressaltar algumas características do funcionamento do Amadeus LMS ${ }^{1}$. O Amadeus permite que o docente disponibilize, no ambiente da disciplina, uma série de recursos, como materiais didáticos direcionados aos alunos, como vídeos, apostilas e atividades. Associados a estes recursos, o Amadeus combina uma série de tags, que descrevem do que se trata o recurso disponibilizado e especificam o assunto discutido no mesmo.

Assim, foi definida como uma das visualizações para o dashboard, uma nuvem de palavras, que indica, pelo tamanho, quais tags obtiveram mais e menos acessos. Ao passar o mouse por cima de cada palavra, aparece para o usuário o número exato de acessos que aquela tag recebeu.

Para os recursos, foi definida uma tabela simples, que mostra todos os recursos disponibilizados na disciplina, e o número de acessos de cada um. Os recursos foram organizados na tabela em ordem decrescente de acessos, ou seja, do mais acessado para o menos acessado.

Além disso, foi disponibilizada no dashboard, uma visualização referente ao engajamento dos alunos na disciplina. Para tanto, foi colocada, ao lado da tabela de recursos, uma nuvem de imagens, que mostra, pelo tamanho da foto do aluno, os alunos que tiveram mais e menos acessos no ambiente da disciplina. Ao passar o mouse sobre a foto de um aluno, o sistema exibe o nome e o número de acessos do aluno. Uma visão geral de todas essas ferramentas é mostrada na Figura 3.

$\mathrm{Na}$ nuvem de imagens, ao clicar na imagem correspondente a um determinado discente, o professor é direcionado para o dashboard do aluno, que já existia no Amadeus LMS, e disponibiliza informações sobre o desempenho do mesmo na disciplina. Os usuários do dashboard conseguem ainda obter informações sobre o desempenho individual de cada

1 https://softwarepublico.gov.br/social/amadeus 
aluno, ao clicar sobre a foto no mesmo, na seção de exibe a nuvem de imagens dos usuários na plataforma.

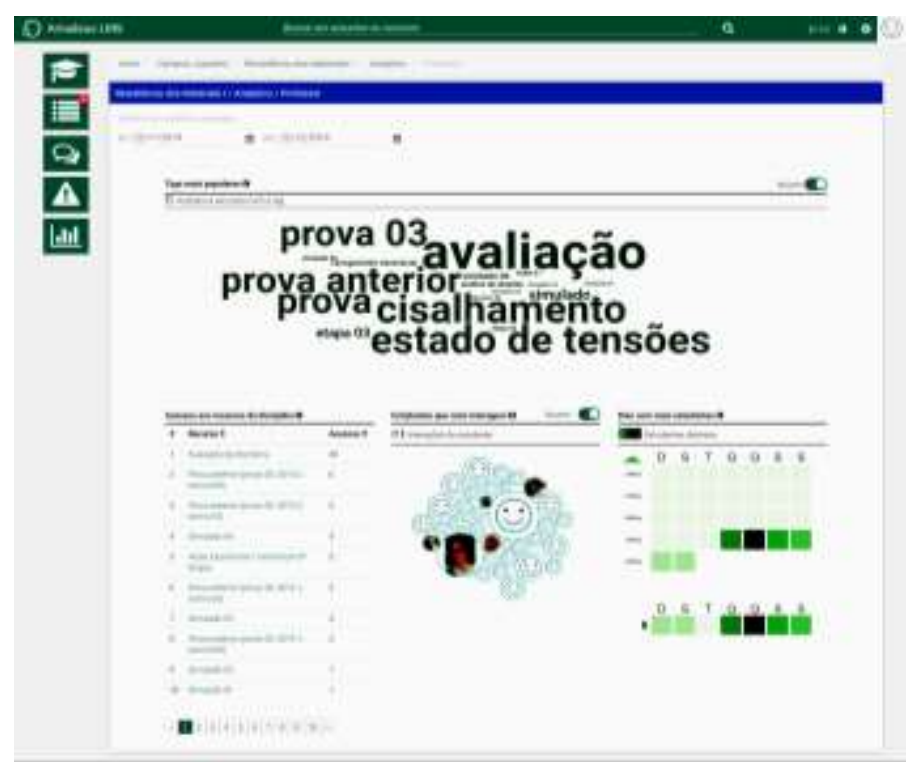

Figura 3- Visão geral do protótipo de alta fidelidade. Fonte: Autores.

A última visualização definida, é um mapa de calor, que mostra, pela intensidade da cor de cada célula, o nível de acessos ao ambiente da disciplina por data, dia da semana e faixa de horários.

\subsection{Resultados da avaliação do dashboard}

Como primeira fonte de evidências da percepção dos usuários sobre a usabilidade do dashboard, foram coletadas as respostas dos participantes da pesquisa ao questionário SUS.

Para análise destes resultados, foram adotados os parâmetros de medida das respostas do SUS, e a classificação dos resultados. A Tabela 1, mostra um resumo das respostas obtidas no questionário de usabilidade.

Tabela 1 - Resultados da aplicação do questionário SUS com os participantes da pesquisa.

\begin{tabular}{|l|c|c|c|c|c|}
\hline \multicolumn{1}{|c|}{ Declaração } & \multicolumn{3}{c|}{$\begin{array}{c}\text { Distribuição de frequência das respostas } \\
\text { em percentual (\%) }\end{array}$} \\
\cline { 2 - 6 } & $\mathbf{1}$ & $\mathbf{2}$ & $\mathbf{3}$ & $\mathbf{4}$ & $\mathbf{5}$ \\
\hline Eu acho que gostaria de utilizar esse recurso frequentemente (D01) & 0 & 0 & 10 & 30 & 60 \\
\hline Eu achei o recurso desnecessariamente complexo (D02) & 100 & 0 & 0 & 0 & 0 \\
\hline Eu achei o recurso fácil de usar (D03) & 0 & 0 & 0 & 10 & 90 \\
\hline $\begin{array}{l}\text { Eu acho que precisaria do apoio de um suporte técnico para usar } \\
\text { esse recurso (D04) }\end{array}$ & 80 & 20 & 0 & 0 & 0 \\
\hline Eu achei que as funções desse recurso foram bem integradas (D05) & 0 & 0 & 0 & 40 & 60 \\
\hline Eu achei que o recurso apresenta muita inconsistência (D06) & 90 & 10 & 0 & 0 & 0 \\
\hline $\begin{array}{l}\text { Eu imagino que a maioria das pessoas aprenderia a usar esse recurso } \\
\text { rapidamente (D07) }\end{array}$ & 0 & 0 & 0 & 0 & 100 \\
\hline Eu achei o recurso muito estranho de usar (D08) & 100 & 0 & 0 & 0 & 0 \\
\hline Eu me senti muito confiante usando esse recurso (D09) & 0 & 0 & 0 & 20 & 80 \\
\hline $\begin{array}{l}\text { Eu precisei aprender uma série de coisas antes que eu pudesse } \\
\text { continuar a utilizar esse recurso (D10) }\end{array}$ & 70 & 30 & 0 & 0 & 0 \\
\hline
\end{tabular}

Os participantes foram unânimes em expressar respostas positivas a respeito do artefato em 9 das 10 questões do SUS. A única questão com discordância, a declaração D01 - "Eu acho que gostaria de utilizar esse recurso frequentemente", ainda possui um percentual elevado de aprovação (90\%). O gráfico mostrado na Figura 4, indica o percentual de cada resposta em cada questão do SUS. 


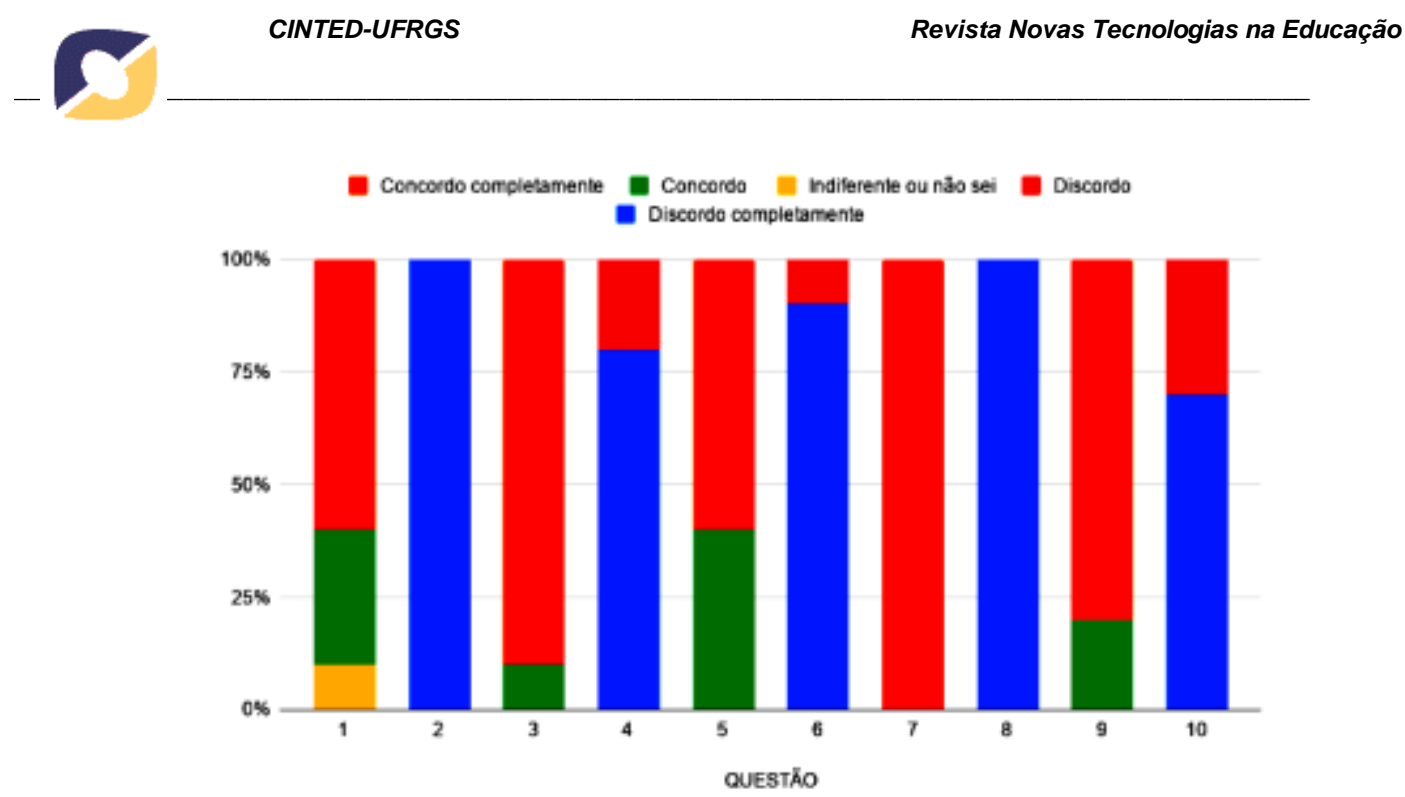

Figura 4 - Proporção percentual de respostas em cada questão do SUS. Fonte: Autores.

O gráfico da Figura 5 mostra a pontuação do SUS, na escala de 0 a 100 [Bangor; Kortum; Miller, 2009], para cada participante da pesquisa. É possível observar, que o participante número 6 marcou uma pontuação igual a 80 no questionário de usabilidade, um valor mais baixo em relação a todos os outros que marcaram mais de 92 pontos, cada um. Sobre o perfil deste participante, podemos considerar dois aspectos que podem ter influenciado em sua avaliação: faixa etária e familiaridade com a ferramenta.

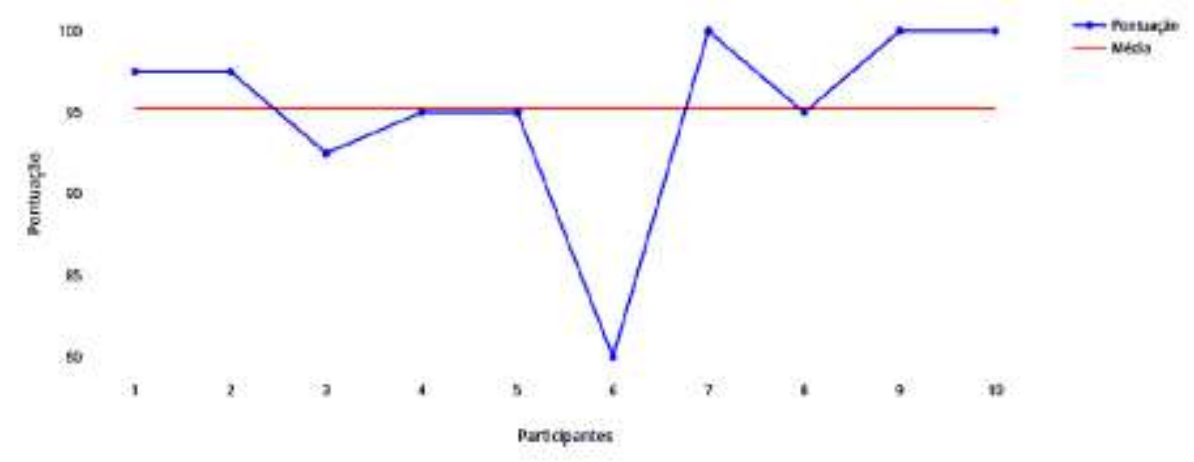

Figura 5 - Pontuação atribuída por cada participante da pesquisa. Fonte: Autores.

Os resultados obtidos em relação à usabilidade do dashboard foram bastante positivos: $90 \%$ dos participantes da pesquisa obtiveram uma pontuação maior que 92 . O participante que mais encontrou dificuldades, como supracitado, obteve uma pontuação igual a 80, que, segundo a classificação de Bangor, Kortum e Miller (2009), ainda corresponde ao nível "bom" de usabilidade.

De acordo com a classificação do SUS, o dashboard obteve uma pontuação média igual a 95,25 sendo classificado como "excelente" do ponto de vista da usabilidade.

\subsection{Resultados do teste de aceitação}

Complementando a análise realizada pela coleta de dados do questionário SUS, são apresentados e discutidos os resultados das questões de aceitação acrescentadas no formulário de avaliação do dashboard. A Tabela 2 mostra um resumo das respostas obtidas nas questões de aceitação.

Há unanimidade de aprovação nas três questões referentes ao visual do dashboard (D04, D05 e D06). Nas demais questões, apesar de haver uma pequena divergência, os resultados se mantêm positivos, com $90 \%$ de aprovação por parte dos entrevistados. O gráfico da Figura 6 mostra a proporção percentual das respostas obtidas em cada questão. 
Tabela 2 - Resultados da aplicação das questões de aceitação do dashboard com os participantes da pesquisa.

\begin{tabular}{|l|c|c|c|c|c|}
\hline \multicolumn{1}{|c|}{ Declaração } & \multicolumn{4}{c|}{$\begin{array}{c}\text { Distribuição de frequência das } \\
\text { respostas em percentual (\%) }\end{array}$} \\
\cline { 2 - 5 } & $\mathbf{1}$ & $\mathbf{2}$ & $\mathbf{3}$ & $\mathbf{4}$ & $\mathbf{5}$ \\
\hline $\begin{array}{l}\text { Os indicadores apresentados podem ser úteis para o acompanhamento das } \\
\text { turmas nas disciplinas que ministro (D01) }\end{array}$ & 0 & 0 & 10 & 30 & 60 \\
\hline $\begin{array}{l}\text { Gostaria de ter acessos aos indicadores presentes no recurso apresentado, } \\
\text { nas disciplinas que ministro (D02) }\end{array}$ & 0 & 0 & 10 & 10 & 80 \\
\hline Eu recomendaria o recurso a outros colegas (D03) & & & & & \\
\hline O recurso possui um visual agradável (D04) & 0 & 0 & 0 & 30 & 70 \\
\hline A quantidade de dados apresentada no recurso foi adequada (D05) & 0 & 0 & 0 & 50 & 50 \\
\hline A organização dos dados apresentados no relatório foi adequada (D06) & 0 & 0 & 0 & 40 & 60 \\
\hline
\end{tabular}

Assim como nos testes de usabilidade, os resultados dos testes de aceitação foram, em sua maioria, positivos.

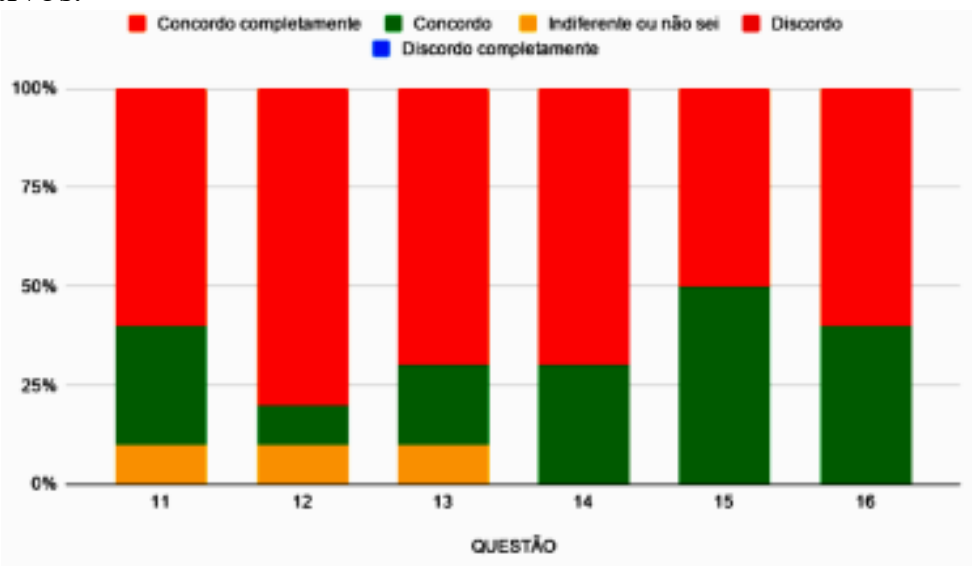

Figura 6 - Proporção percentual de respostas em cada de aceitação do dashboard. Fonte: Autores.

Os resultados demonstraram a boa usabilidade e a elevada aceitação do dashboard proposto, o que indica que o dashboard pode ser incorporado como mais um recurso no Amadeus LMS, agregando um importante recurso para Learning Analytics.

\section{Considerações Finais e Trabalhos Futuros}

Este trabalho apresentou uma proposta de solução em Learning Analytics focada no contexto da abordagem Blended Learning. A solução proposta, consistiu em um dashboard direcionado para o uso de professores, no ambiente acadêmico. O dashboard apresenta informações referentes às interações dos alunos no ambiente da disciplina.

A fim de contribuir no âmbito da computação, incentivar o compartilhamento de projetos desta natureza e fortalecer o campo de informática na educação, o artefato proposto já foi implementado como uma solução para o Amadeus LMS, um software livre, registrado sob licença de Software Público Brasileiro. A avaliação desse artefato foi realizada com professores do ensino superior, tendo em vista a análise da efetividade de um artefato de Learning Analytics no acompanhamento de atividades em cursos Blended Learning.

Por ser classificada como uma pesquisa do tipo Design Science, utilizou-se a metodologia proposta para pesquisas desta categoria, a Design Science Research, cujo método de 6 etapas foi fundamental para a organização e execução do trabalho.

Os objetivos da pesquisa foram atingidos de forma satisfatória. $O$ planejamento realizado com a revisão de literatura, análise de trabalhos relacionados e desenvolvimento dos protótipos, mostrou-se bastante eficiente, uma vez que o resultado final foi bem avaliado, tanto em usabilidade, quanto em aceitação, pelos participantes da pesquisa. 
Trabalhos futuros podem ser realizados a fim de melhorar a qualidade dos indicativos apresentados no dashboard, podendo, inclusive, acrescentar algumas das sugestões propostas pelos participantes da pesquisa.

\section{Referências}

BANGOR, A.; KORTUM, P.; MILLER, J. Determining what individual sus scores mean: Adding an adjective rating scale. Journal of usability studies, Usability Professionals' Association, v. 4, n. 3, p. 114-123, 2009.

BAX, M. P. Design science: filosofia da pesquisa em ciência da informação e tecnologia. 2017.

BROOKE, J. et al. Sus-a quick and dirty usability scale. Usability evaluation in industry, London-, v. 189, n. 194, p. 4-7, 2013.

CHATTI, M. A. et al. A reference model for Learning Analytics. International Journal of Technology Enhanced Learning, Inderscience Publishers Ltd., PO Box 735 Olney Bucks MK 465 WB United Kingdom, v. 4, n. 5-6, p. 318-331, 2013.

CLOW, Doug. The Learning Analytics cycle: closing the loop effectively. In: Proceedings of the 2nd international conference on Learning Analytics and knowledge. 2012. p. 134-138.

CORRIN, L. et al. Loop: A Learning Analytics tool to provide teachers with useful data visualisations. Perth: Ascilite, 2015.

DAVIS, F. D.; BAGOZZI, R. P.; WARSHAW, P. R. User acceptance of computer technology: a comparison of two theoretical models. Management science, INFORMS, v. 35, n. 8, p. 982-1003, 1989.

DUMAS, J. S.; REDISH, J. A practical guide to usability testing. [S.1.]: Intellect books, 1999.

DUVAL, E. Attention please!: Learning Analytics for visualization and recommendation. LAK, v. 11, p. $9-17,2011$

EINHARDT, L.; TAVARES, T. A.; CECHINEL, C. Moodle analytics dashboard: A Learning Analytics tool to visualize users interactions in moodle. In: IEEE. 2016 XI Latin American Conference on Learning Objects and Technology (LACLO). [S.1.], 2016. p. 1-6.

GOMES, C. V. C.; Proposta de um Dashboard para Ampliar a Percepção dos Professores Sobre as Atividades se Estudantes em Ambiente Virtual de Aprendizagem. Trabalho de Conclusão de Curso (Graduação em Engenharia de Computação). UNIVASF. Juazeiro. 2020.

PEFFERS, K. et al. A design science research methodology for information systems research. Journal of management information systems, Taylor \& Francis, v. 24, n. 3, p. 45-77, 2007.

SIEMENS, G. et al. Open Learning Analytics: an integrated \& modularized platform:Society for Learning Analytics research. Open Learning Analytics. Retrieved from http://solaresearch. org/initiatives/ola, 2011.

SILVA, J. C. S. Uma abordagem de Learning Analytics para a autorregulação da aprendizagem de estudantes em sala de aula invertida. Tese de Doutorado. Universidade Federal de Pernambuco, 2018.

SILVA, J. et al. Uma abordagem para integração do moodle com o framework shiny para Learning Analytics. In: Anais dos Workshops do Congresso Brasileiro de Informática na Educação. [S.l.: s.n.], 2016. v. 5, n. 1, p. 930.

TORI, R. Cursos híbridos ou blended learning. Litto FM, Formiga MMM. Educação a distância: o estado da arte, v. 1, p. 121-128, 2009.

VOZNIUK, A.; GOVAERTS, S.; GILLET, D. Towards portable Learning Analytics dashboards. In: IEEE. 2013 IEEE 13th International Conference on Advanced Learning Technologies. [S.1.], 2013. p. $412-416$.

XHAKAJ, F.; ALEVEN, V.; MCLAREN, B. M. Effects of a teacher dashboard for an intelligent tutoring system on teacher knowledge, lesson planning, lessons and student learning. In: SPRINGER. European Conference on Technology Enhanced Learning. [S.1.], 2017. p. 315-329. 